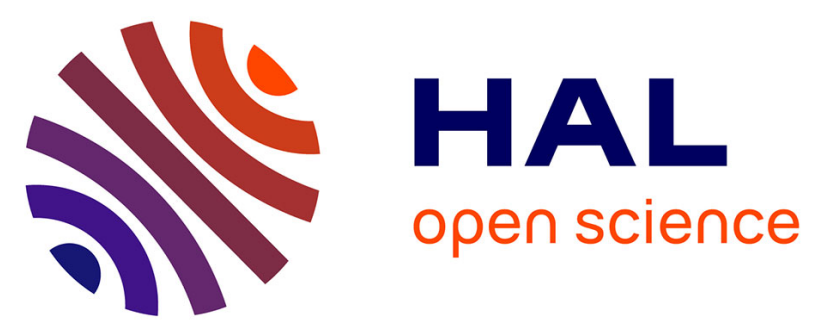

\title{
New Formulation of the Equations Describing the Locked States of Two Van der Pol Oscillators Coupled through a Broadband Network - Application to the Design of Two Differential Coupled VCOs
}

David Cordeau, Mihaela-Izabela Ionita, Jean-Marie Paillot, Mihai Iordache

\section{To cite this version:}

David Cordeau, Mihaela-Izabela Ionita, Jean-Marie Paillot, Mihai Iordache. New Formulation of the Equations Describing the Locked States of Two Van der Pol Oscillators Coupled through a Broadband Network - Application to the Design of Two Differential Coupled VCOs. Frequenz Journal of RFEngineering and Telecommunications, 2013, 67 (7-8), pp.237-247. 10.1515/freq-2012-0089 . hal00821885

\section{HAL Id: hal-00821885 \\ https://hal.science/hal-00821885}

Submitted on 13 May 2013

HAL is a multi-disciplinary open access archive for the deposit and dissemination of scientific research documents, whether they are published or not. The documents may come from teaching and research institutions in France or abroad, or from public or private research centers.
L'archive ouverte pluridisciplinaire HAL, est destinée au dépôt et à la diffusion de documents scientifiques de niveau recherche, publiés ou non, émanant des établissements d'enseignement et de recherche français ou étrangers, des laboratoires publics ou privés. 


\title{
NEW FORMULATION OF THE EQUATIONS DESCRIBING THE LOCKED STATES OF TWO VAN DER POL OSCILLATORS COUPLED THROUGH A BROADBAND NETWORK - APPLICATION TO THE DESIGN OF TWO DIFFERENTIAL COUPLED VCOS
}

\author{
David Cordeau ${ }^{1}$, Mihaela-Izabela Ionita ${ }^{2}$, Jean-Marie Paillot ${ }^{1}$ and Mihai Iordache ${ }^{2}$ \\ ${ }^{1}$ University of Poitiers-CNRS-XLIM UMR 7252, IUT, 4 avenue de Varsovie, 16021 Angoulême, France \\ ${ }^{2}$ Electrical Engineering Faculty, University Politehnica of Bucharest, Bucharest, Romania \\ e-mail :david.cordeau@univ-poitiers.fr, mihaelaizabela@yahoo.com, jean.marie.paillot@univ-poitiers.fr, iordache@elth.pub.ro
}

Shortened title: Synchronization of oscillators coupled through a broadband network

\begin{abstract}
In this paper, a new expression of the equations describing the locked states of two van der Pol oscillators coupled through a broadband network and allowing an accurate prediction of the oscillators' amplitudes is presented. To do so, the York's theory is improved using a more accurate van der Pol model in order to obtain a new system of four equations with four unknowns describing the locked states of two van der Pol oscillators coupled through a resonant network. Then, the case of a resistive coupling circuit is deduced from this new system of equations. The theory regarding the prediction of the oscillators' amplitudes is verified using Agilent's ADS software for the practical case of an array made of two differential Voltage Controlled Oscillators (VCOs) coupled through a resistive network.
\end{abstract}

Keywords: Coupled oscillators, resistive coupling network, synchronization, van der Pol oscillator, Voltage Controlled Oscillators.

\section{Introduction}

Arrays of coupled oscillators offer a potentially useful technique for producing higher powers at millimeterwave frequencies with better efficiency than is possible with conventional power-combining techniques [1,2]. Another application is the beam steering of antenna arrays [3], through a constant phase progression in the oscillator chain which is obtained by detuning the free-running frequencies of the outermost oscillators in the array $[4,5]$. Moreover, it is shown that the resulting inter-stage phase shift is independent of the number of oscillators in the array $[6,7,8]$. Furthermore, synchronization phenomena in arrays of coupled oscillators are very important models to describe various higher-dimensional nonlinear phenomena in the field of natural science [9].

Many techniques have been used to analyse the behaviour of coupled oscillators for many years such as time domain approaches [10-15]. Concerning the frequency domain approaches, R. York made use of simple van der Pol oscillators to model microwave oscillators coupled through either a resistive network or a broadband network $[6,16,17]$. Since these works are limited to cases where the coupling network bandwidth is much greater than the oscillators' bandwidth, he used more accurate approximations based on a generalization of Kurokawa's method [18] to extend the study to the case of a narrow-band circuit [19]. This theory allows the equations for the amplitude and phase dynamics of two oscillators coupled through many types of circuits to be derived. Since these works, only few papers present new techniques for the analysis of coupledoscillator arrays in the frequency domain [20-23]. In [22], a semi-analytical formulation is presented for the design of coupled-oscillator systems, avoiding the computational expensiveness of a full harmonic balance 
synthesis presented in [20] and [21]. In [24], a simplified closed-form of the semi-analytical formulation proposed in [22] for the optimised design of coupled-oscillator systems is presented. Nevertheless, even if this new semi-analytical formulation allows a good prediction of the coupled-oscillator solution, it is only valid for the weak coupling case whereas the York's theory [19] provides a full analytical formulation allowing to predict the performances of microwave oscillator arrays for both weak and strong coupling. Unfortunately, although this latter theory provides simple approximate formulas for the dimensions of the locking region, for the amplitudes of the two coupled oscillators and for the inter-stage phase shift, the van der Pol model used is too simple and doesn't allow an accurate prediction of the amplitudes.

Furthermore, since the theoretical limit of the phase shift obtained for an array of single-ended coupled oscillators is within the range of $\pm 90^{\circ}$ [6], it seems to be interesting to analyse the behaviour of an array of coupled differential VCOs. Indeed, in this case, the theoretical limit of the phase shift is within $360^{\circ}$ due to the differential nature of the array. In these conditions, a continuously controlled $360^{\circ}$ phase shifting range can be obtained leading to a more efficient beam-scanning architecture for instance. Furthermore, differential VCOs are widely used in high-frequency circuit design due to their relatively good phase noise performances and ease of integration. Moreover, the use of a broadband coupling network, i.e. a resistor, instead of a resonant one can lead to a substantial save in chip area.

Due to these considerations, the aim of this paper is to present a new formulation of the equations describing the locked states of two van der Pol oscillators coupled through a broadband network using an accurate model allowing a good prediction of the oscillators' amplitudes. The accuracy of the theory will be validated by simulations for the practical case of two NMOS differential VCOs coupled through a resistive network. To do so, an overview of the equations developed by R. York giving the dynamics for two van der Pol oscillators coupled through a resonant network will be provided in section 2 . The details concerning the van der Pol model used in [19] will be given showing the limitations of such an approach. In section 3, a new expression of the York's equations using a more accurate van der Pol model will be presented. Then, the case of a resistive coupling circuit will be treated. In section 4, the theory is verified using Agilent's software ADS for an array made of two differential VCOs coupled through a resistive network.

\section{Overview of the dynamics of two van der Pol oscillators coupled through a resonant network}

The theory elaborated by J. Lynch and R. York in [19] is exemplified using an architecture made of two parallel resonant circuits containing nonlinear negative conductance devices and coupled through a series resonant circuit, as shown in Fig. 1. These oscillators are considered identical, except for their free-running frequencies. Thus, starting from the admittance transfer functions $\left(Y_{1}, Y_{2}, Y_{\mathrm{c}}\right)$ binding the coupling current $\left(I_{\mathrm{c}}\right)$ to the oscillators voltages $\left(V_{1}\right.$ and $\left.V_{2}\right)$, and relying on Kurokawa's substitution [18], the authors cited previously described the oscillators dynamic equations, as well as those for the amplitude and phase of the coupling current. Then, by setting the derivatives equal to zero, the algebraic equations describing the oscillators' frequency locked states were obtained. This system of four equations with four unknowns $\left(A_{1}, A_{2}\right.$, $\Delta \phi$ and $\omega$ ) is presented as follows:

$$
\begin{gathered}
\left(1-\lambda_{o} \varepsilon^{2}-A_{1}^{2}\right) A_{1}=-\lambda_{o} \varepsilon A_{2} \cos (\Delta \phi-\Phi) \\
\Delta \omega_{o 1}-\left(1-\lambda_{o} \varepsilon^{2} \frac{\omega_{a}}{\omega_{a c}}\right) \Delta \omega_{c}=-\lambda_{o} \varepsilon \omega_{a} \frac{A_{2}}{A_{1}} \sin (\Delta \phi-\Phi) \\
\left(1-\lambda_{o} \varepsilon^{2}-A_{2}^{2}\right) A_{2}=-\lambda_{o} \varepsilon A_{1} \cos (\Delta \phi+\Phi) \\
\Delta \omega_{o 2}-\left(1-\lambda_{o} \varepsilon^{2} \frac{\omega_{a}}{\omega_{a c}}\right) \Delta \omega_{c}=\lambda_{o} \varepsilon \omega_{a} \frac{A_{1}}{A_{2}} \sin (\Delta \phi+\Phi) .
\end{gathered}
$$


With

- $\lambda_{o}=\frac{1}{G_{0} R_{c}}$ : the coupling constant, where $G_{0}$ is the nonlinear device conductance at zero voltage ;

- $2 \omega_{a}=\frac{G_{0}}{C}$ : the oscillators bandwidth ;

- $2 \omega_{a c}=\frac{R_{c}}{L_{c}}:$ the unloaded coupling circuit bandwidth ;

- $\quad A_{1}, A_{2}$ : the amplitudes of oscillators 1 and 2, respectively ;

- $\Delta \phi$ : the inter-stage phase shift ;

- $\omega_{o 1}, \omega_{o 2}$ : the free-running frequencies or tunings of oscillators 1 and 2, respectively ;

- $\omega_{o c}$ : resonant frequency of the coupling circuit ;

- $\varepsilon=\frac{1}{\sqrt{\left(\Delta \omega_{c}\right)^{2}}}=\cos (\Phi)$ : the coupling strength scale factor ;

$$
\sqrt{1+\left(\frac{\Delta \omega_{c}}{\omega_{a c}}\right)^{2}}
$$

- $\Phi=\tan ^{-1}\left(\frac{\Delta \omega_{c}}{\omega_{a c}}\right):$ the coupling phase.

Furthermore, the free-running frequencies of the oscillators, $\omega_{o 1}$ and $\omega_{o 2}$, and the synchronization frequency of the system, $\omega$, are referred to the resonant frequency of the coupling circuit, $\omega_{o c}$, using the following substitution :

$$
\Delta \omega_{o 1}=\omega_{o 1}-\omega_{o c} \quad \Delta \omega_{o 2}=\omega_{o 2}-\omega_{o c} \quad \Delta \omega_{c}=\omega-\omega_{o c}
$$

Thus, a solution to (1) indicates the existence of a frequency-locked state and provides the user with the amplitudes $A_{1}$ and $A_{2}$ of the two oscillators as well as the inter-stage phase shift $\Delta \phi$ and the synchronization pulsation $\omega$ for a combination $\left(\Delta \omega_{01}, \Delta \omega_{02}\right)$.

Nevertheless, to simplify the analysis, J. Lynch and R. York made use of cases of practical interest according to the tunings of oscillators 1 and 2. Indeed, for equal tunings, i.e. for $\Delta \omega_{01}=\Delta \omega_{02}$, one can show, using the second and fourth equations of (1), that the phase difference $\Delta \phi$ equals zero and the oscillators will lock on a bandwidth depending on the value of the coupling circuit bandwidth $\left(\omega_{\mathrm{ac}}\right)$ with equal amplitudes $\left(A_{1}=A_{2}\right)$ [19].

\section{Coupling circuit}

$$
\mathrm{L}_{\mathrm{c}} \quad \mathrm{C}_{\mathrm{c}} \quad \mathrm{R}_{\mathrm{c}}
$$

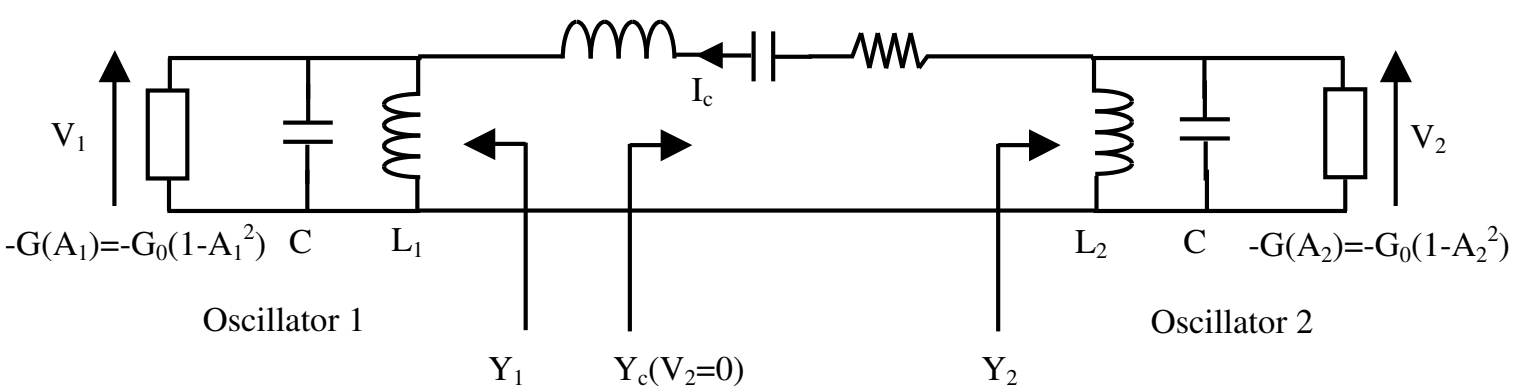

Figure 1: Two oscillators coupled through a resonant circuit 
On the other hand, when the coupling circuit resonance $\left(\omega_{o c}\right)$ is located exactly between the two oscillators free-running frequencies, i.e. for $\Delta \omega_{01}=-\Delta \omega_{02}$, one can show, again using the second and fourth equations of (1), that $\Delta \omega_{c}=0$, which implies $\Phi=0$ and $A_{1}=A_{2}$. In these conditions, the frequency difference between the two free-running frequencies of oscillators 1 and 2 can be expressed, by subtracting the fourth and second equations, as follows:

$$
\Delta \omega_{0}=2 \lambda_{0} \omega_{a} \sin (\Delta \phi)
$$

with $\Delta \omega_{0}=\omega_{02}-\omega_{01}$ as mentioned previously.

Furthermore, in the same conditions, the amplitudes are equal and found from (1), by combining the first and third equation, as follows:

$$
A^{2}=1-\lambda_{0}\left(1-\sqrt{1-\left(\frac{\Delta \omega_{0}}{2 \lambda_{0} \omega_{a}}\right)^{2}}\right)
$$

As explained in [19], the amplitude will remain close to the unity for weak coupling, i.e. when $\lambda_{0}<<\frac{1}{2}$. Nevertheless, for weak coupling, the amplitudes of the oscillators will remain close to their free-running values. Thus, with the van der Pol model used in this theory, the free-running amplitudes of the oscillators are always equal to unity showing the limitations of this theory regarding the prediction of the amplitudes of the two coupled oscillators.

To illustrate this affirmation, let us explain the assumptions and approximations made by J. Lynch and R. York for the modelling of a van der Pol oscillator. Indeed, let us first consider the schematic of a van der Pol oscillator as shown in Fig. 2 for which the current in the nonlinear conductance $G_{\mathrm{NL}}$ is equal to $i_{1}(t)=-a v(t)+b v^{3}(t)$ with $-a$ the negative conductance necessary to start the oscillations and $b$ a parameter used to model the saturation phenomenon. In this case, according to the Kirchhoff's current law, the current $i(\mathrm{t})$ can be written as

$$
i(t)=(-a+G) \cdot v(t)+b \cdot v^{3}(t)
$$

$\mathrm{i}(\mathrm{t})$

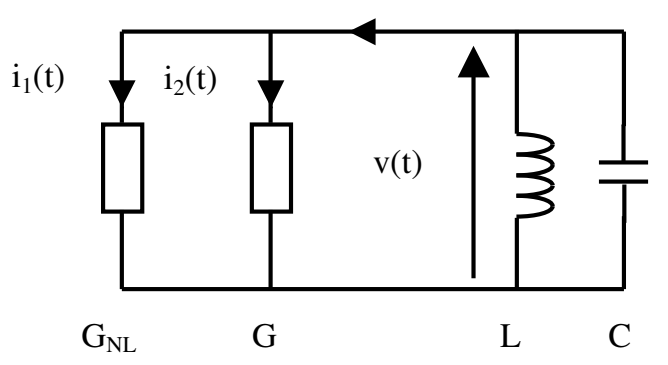

Figure 2 : van der Pol oscillator model

Now, using the assumption of a perfectly sinusoidal oscillation so that $v(t)=A \cdot \cos \left(\omega_{0} t\right)$, from (4) we have

$$
i(t)=\left(-a+G+\frac{3}{4} b A^{2}\right) \cdot A \cdot \cos \left(\omega_{0} t\right)+b \frac{A^{3}}{4} \cos \left(3 \omega_{0} t\right) .
$$


Furthermore, let us recall that such an oscillator topology can be modelled by a quasi-linear representation allowing a very simple analytical calculation [25]. In this case, the expression in the first bracket in (5) represents the negative conductance presented by the active part. Nevertheless, in York's model [19], this negative conductance is equal to

$$
-G(A)=-G_{0}\left(1-A^{2}\right)
$$

In these conditions, the expression in the first bracket in (5) and (6) leads to

$$
G_{0}=a-G=\frac{3}{4} b
$$

This result clearly shows the limitations of the van der Pol model used by Lynch and York in [19] since this model is valid only for van der Pol parameters values $a$ and $b$ for which (7) is fulfilled. Furthermore, one can show that, in this case, the free-running amplitude of the van der Pol oscillator is always equal to unity. Indeed, the condition of free-running oscillation of one of the oscillators of Fig. 1 at a frequency $f_{0}$ can be written as

$$
Y_{N L}\left(A, \omega_{0}\right)+Y_{L}\left(\omega_{0}\right)=0 .
$$

where the subscripts " $N L "$ and " $L "$ denote the nonlinear and linear portions of the circuit and with $Y_{N L}\left(A, \omega_{0}\right)=-G(A)=-G_{0}\left(1-A^{2}\right)$ and $Y_{L}=j\left(C \omega-\frac{1}{L \omega}\right)$

Thus, according to (8), the frequency of oscillations is found to be $f_{0}=\frac{1}{2 \pi \sqrt{L C}}$ and the amplitude is $A=1$.

As a consequence, a new formulation of (1) using a more accurate van der Pol model will be presented in the next section. The case of a resistive coupling circuit will then be deduced from the obtained system of equations.

\section{New expression of the equations describing the locked states of two van der Pol coupled oscillators}

\subsection{Two van der Pol oscillators coupled through a resonant network}

In order to obtain the new system of four equations with four unknowns describing the locked states of two van der Pol oscillators coupled through a resonant network, the theory of J. Lynch and R. York [19] will be adapted to the case of a more accurate van der Pol model. To do so, let us consider the circuit of Fig. 3 made of two van der Pol oscillators coupled through a series RLC circuit. These oscillators are considered identical, except for their free-running frequencies. In this case, the frequency-domain equations, using the admittance transfer function binding the coupling current to the oscillators voltages, can be written as follows:

$$
\begin{gathered}
I_{c}=Y_{1}\left(A_{1}, \omega_{1}\right) \cdot V_{1}=\left[-G_{N L}\left(A_{1}\right)+Y_{L 1}\left(\omega_{1}\right)\right] \cdot V_{1} \\
I_{c}=-Y_{2}\left(A_{2}, \omega_{2}\right) \cdot V_{2}=-\left[-G_{N L}\left(A_{2}\right)+Y_{L 2}\left(\omega_{2}\right)\right] \cdot V_{2} \\
I_{c}=-Y_{c}\left(\omega_{c}\right) \cdot\left(V_{1}-V_{2}\right) .
\end{gathered}
$$

where $A_{1}, \omega_{1}$, represents the amplitude and pulsation of oscillator 1 , etc... and the subscripts " $N L$ " and " $L$ " denote the nonlinear and linear portions of the circuit. Let us now determine the oscillator admittance function for oscillator 1 which can be written as follows: 


$$
Y_{1}\left(A_{1}, \omega_{1}\right)=-G_{N L}\left(A_{1}\right)+G_{L}+\frac{C}{j \omega_{1}}\left(\omega_{01}^{2}-\omega_{1}^{2}\right)
$$

where $\omega_{01}=\frac{1}{\sqrt{L_{1} C}}$ is the tank resonant frequency of oscillator 1 and $-G_{N L}\left(A_{1}\right)=-a+\frac{3}{4} b A_{1}^{2}$ is the negative conductance presented by the active part as explained in (5) leading to sinusoidal oscillation. Now, if the frequency of oscillator 1 remains close to its "free running" frequency or uncoupled value $\omega_{01}$, then, the admittance function can be written as

$$
Y_{1}\left(A_{1}, \omega_{1}\right) \cong G_{L}\left(1-\frac{G_{N L}\left(A_{1}\right)}{G_{L}}\right)+\frac{2 C}{j}\left(\omega_{01}-\omega_{1}\right) .
$$

Now, let us note $2 \omega_{a}=\frac{G_{L}}{C}$ which represents the resonator "bandwidth" and using the substitution $\frac{G_{N L}\left(A_{1}\right)}{G_{L}}-1=S\left(A_{1}\right)$ in order to simplify the notation, we have

$$
Y_{1}\left(A_{1}, \omega_{1}\right) \cong-G_{L}\left[S\left(A_{1}\right)+j \frac{\left(\omega_{01}-\omega_{1}\right)}{\omega_{a}}\right] .
$$

In the same way, the admittance function for oscillator 2 is found to be

$$
Y_{2}\left(A_{2}, \omega_{2}\right) \cong-G_{L}\left[S\left(A_{2}\right)+j \frac{\left(\omega_{02}-\omega_{2}\right)}{\omega_{a}}\right] .
$$

Furthermore, let us note that the admittance functions for oscillators 1 and 2 are similar to those obtained by Lynch and York in [19] except that $G_{L}$ and $S\left(A_{i}\right)$ replace $G_{0}$ and $f\left(A_{i}\right)=1-A_{i}^{2}$. Nevertheless, let us recall that a more accurate van der Pol model is used in (12) and (13) since $S\left(A_{i}\right)=\frac{G_{N L}\left(A_{i}\right)}{G_{L}}-1$ with $-G_{N L}\left(A_{i}\right)=-a+\frac{3}{4} b A_{i}^{2}$.

\section{Coupling circuit}

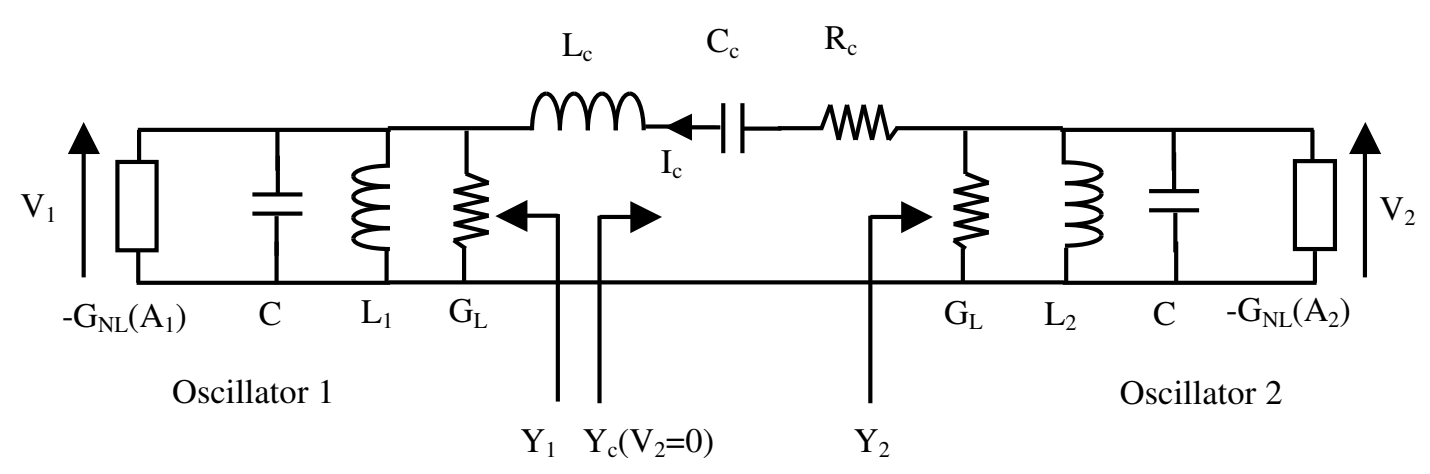

Figure 3: Two van der Pol oscillators coupled through a series RLC circuit 
Thus, following the same steps as in [19] but using (12) and (13) for the admittance functions of oscillators 1 and 2 and replacing $G_{0}$ by $G_{L}$, the new system of four equations with four unknowns describing the locked states of two van der Pol oscillators coupled through a resonant network is the following:

$$
\begin{aligned}
& \left(S\left(A_{1}\right)-\lambda_{o} \varepsilon^{2}\right) A_{1}=-\lambda_{o} \varepsilon A_{2} \cos (\Delta \phi-\Phi) \\
& \Delta \omega_{o 1}-\left(1-\lambda_{o} \varepsilon^{2} \frac{\omega_{a}}{\omega_{a c}}\right) \Delta \omega_{c}=-\lambda_{o} \varepsilon \omega_{a} \frac{A_{2}}{A_{1}} \sin (\Delta \phi-\Phi) \\
& \left(S\left(A_{2}\right)-\lambda_{o} \varepsilon^{2}\right) A_{2}=-\lambda_{o} \varepsilon A_{1} \cos (\Delta \phi+\Phi) \\
& \Delta \omega_{o 2}-\left(1-\lambda_{o} \varepsilon^{2} \frac{\omega_{a}}{\omega_{a c}}\right) \Delta \omega_{c}=\lambda_{o} \varepsilon \omega_{a} \frac{A_{1}}{A_{2}} \sin (\Delta \phi+\Phi) \text {. } \\
& \text { where } \lambda_{0}=\frac{1}{G_{L} R_{c}}, 2 \omega_{a}=\frac{G_{L}}{C} \text { and } S\left(A_{i}\right)=\frac{G_{N L}\left(A_{i}\right)}{G_{L}}-1=\frac{a-\frac{3}{4} b A_{i}^{2}-G_{L}}{G_{L}}
\end{aligned}
$$

Thus, for the specific case of $\Delta \omega_{01}=-\Delta \omega_{02}$ for which the coupling circuit resonance is located exactly between the two oscillators free-running frequencies, one can show, using (14), that $\Delta \omega_{c}=0$, which implies $\Phi=0, \varepsilon=1$ and $A_{1}=A_{2}=A$. As a consequence, the frequency difference between the two free-running frequencies of oscillators 1 and 2 can also be expressed using (2) but with $\lambda_{0}=\frac{1}{G_{L} R_{c}}$ and $\omega_{a}=\frac{G_{L}}{2 C}$. Nevertheless, since, in these conditions, the amplitudes are equal, the following condition needs to be satisfied:

$$
S(A)-\lambda_{0}=-\lambda_{0} \cos (\Delta \phi)
$$

Now replacing $S(A)$ by its expression we have

$$
\frac{a-\frac{3}{4} b A^{2}}{G_{L}}-1-\lambda_{0}=-\lambda_{0} \cos (\Delta \phi)
$$

and then

$$
A=\sqrt{\frac{4}{3 b} \cdot\left[a-G_{L}\left(1-\lambda_{0}(\cos (\Delta \phi)-1)\right)\right]}
$$

One can notice that this expression is different from the one obtained in (3) and allows the prediction of the amplitudes of the two coupled van der Pol oscillators for values of parameters $a$ and $b$ of the van der Pol non linearity leading to a sinusoidal waveform.

\subsection{Resistive coupling circuit case}

The case of a resistive coupling circuit is found by letting the coupling circuit bandwidth approach infinity so that $\omega_{a c} \rightarrow \infty$ leading to $\varepsilon=1$ and $\Phi=0$ according to (1). In these conditions, the new equations describing the locked states can be deduced from (14) as follows : 


$$
\begin{gathered}
\left(S\left(A_{1}\right)-\lambda_{o}\right) A_{1}=-\lambda_{o} A_{2} \cos (\Delta \phi) \\
\Delta \omega_{o 1}-\Delta \omega_{c}=-\lambda_{o} \omega_{a} \frac{A_{2}}{A_{1}} \sin (\Delta \phi) \\
\left(S\left(A_{2}\right)-\lambda_{o}\right) A_{2}=-\lambda_{o} A_{1} \cos (\Delta \phi) \\
\Delta \omega_{o 2}-\Delta \omega_{c}=\lambda_{o} \omega_{a} \frac{A_{1}}{A_{2}} \sin (\Delta \phi)
\end{gathered}
$$

Since $\Delta \omega_{01}-\Delta \omega_{c}=\omega_{01}-\omega$ and $\Delta \omega_{02}-\Delta \omega_{c}=\omega_{02}-\omega$

Thus, we have

$$
\begin{gathered}
\left(S\left(A_{1}\right)-\lambda_{o}\right) A_{1}=-\lambda_{o} A_{2} \cos (\Delta \phi) \\
\omega_{o 1}=\omega-\lambda_{o} \omega_{a} \frac{A_{2}}{A_{1}} \sin (\Delta \phi) \\
\left(S\left(A_{2}\right)-\lambda_{o}\right) A_{2}=-\lambda_{o} A_{1} \cos (\Delta \phi) \\
\omega_{o 2}=\omega+\lambda_{o} \omega_{a} \frac{A_{1}}{A_{2}} \sin (\Delta \phi) .
\end{gathered}
$$

Hence, knowing the parameters $\lambda_{0}, \omega_{\mathrm{a}}, a$, and $b$, a solution to the system (16) provides the user with the amplitudes $A_{1}$ and $A_{2}$ of the two oscillators as well as the inter-stage phase shift $\Delta \phi$ and the synchronization pulsation $\omega$ for a combination $\left(\omega_{01}, \omega_{02}\right)$. Nevertheless, since the two oscillators are considered identical and for a broadband or resistive coupling circuit, the synchronization frequency is expected to be located between the two oscillators' free-running frequencies [19], so that:

$$
\omega=\frac{\omega_{01}+\omega_{02}}{2}
$$

Thus, according to the second and fourth equations of (16), this implies $A_{1}=A_{2}$. In these conditions, the form of the phase difference $\Delta \phi$ is found again from (16) by subtracting the fourth and second equations leading to the same equation as (2) with $\lambda_{0}=\frac{1}{G_{L} R_{c}}$ and $\omega_{a}=\frac{G_{L}}{2 C}$.

Furthermore, the amplitudes, which are equal in this case, are found from (16) by combining the first and third equation leading to exactly the same result as in (15).

\section{Simulations and verification}

In order to verify the accuracy of the presented theory regarding the prediction of the oscillators' amplitudes, simulations using Agilent's ADS software will be performed for the practical case of two identical differential VCOs coupled through a resistor $R_{c} / 2$ equal to $260 \Omega$, as shown in Fig. 4. The VCO structure is based on a cross-coupled NMOS differential topology using a $0.35 \mu \mathrm{m}$ BiCMOS SiGe process. The cross connected NMOS differential pair provides the negative resistance to compensate for the tank losses and the tail current source is a simple NMOS current mirror which draws $12 \mathrm{~mA}$ under $\mathrm{Vcc}=2.5 \mathrm{~V}$. 


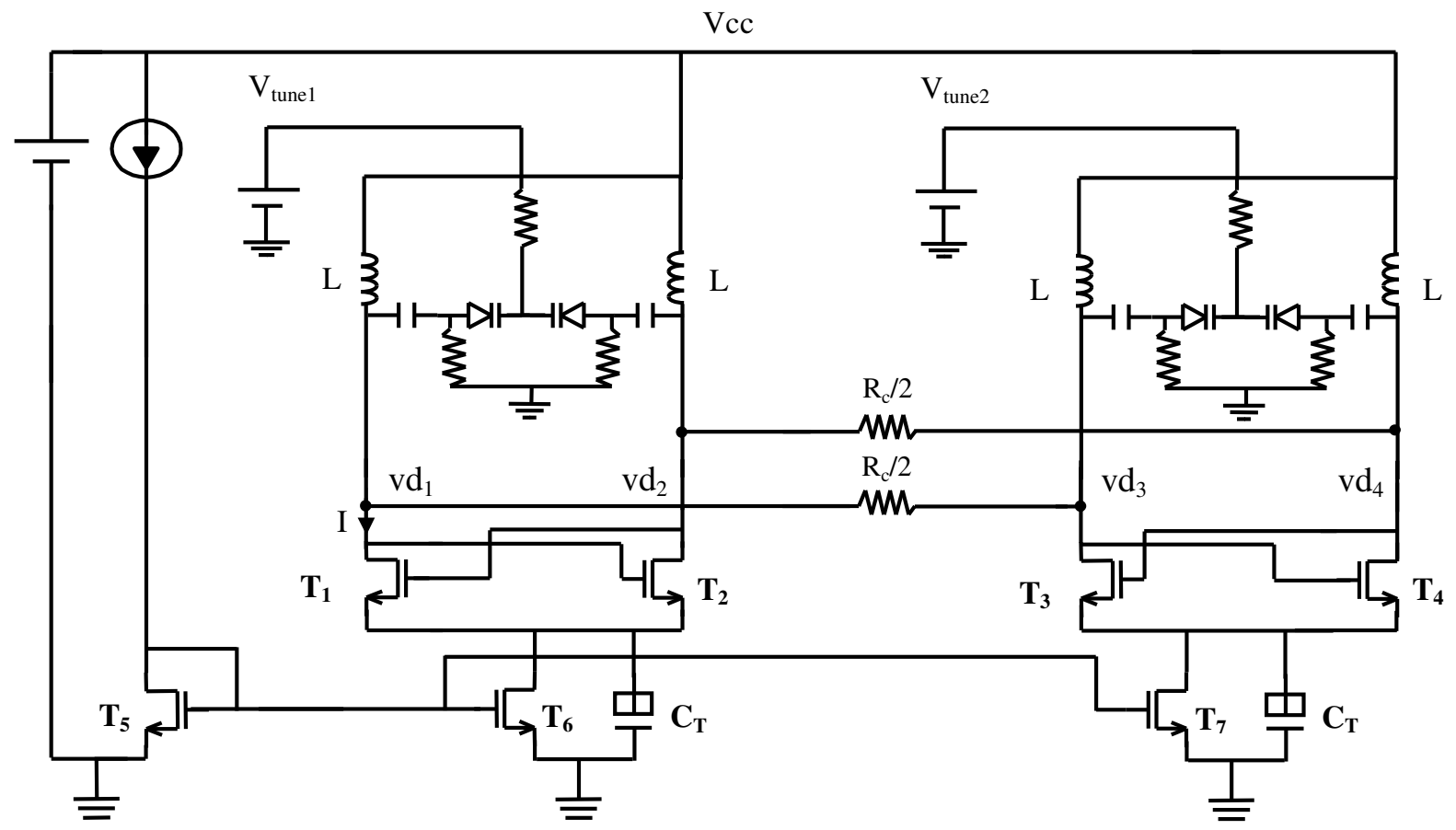

Figure 4: Two differential VCOs coupled through a resistor

The frequency of oscillation is determined by the $L C$ tank at the drains and is chosen to be close to $6 \mathrm{GHz}$ with an inductance value, $L$, close to $0.8 \mathrm{nH}$ with a quality factor, $Q$, equal to 15 at $6 \mathrm{GHz}$. The tuning range depends on the global capacitance $C$ variation and thus on the $C_{\max } / C_{\min }$ ratio of the $\mathrm{P}+/ \mathrm{N}$ varactor diodes and on the AC coupling capacitor.

A tail capacitor $C_{T}$ is used to attenuate both the high-frequency noise component of the tail current and the voltage variations on the tail node [26].

Furthermore, to ensure a proper start-up of the VCO, the following condition needs to be satisfied:

$$
g_{m}>\frac{1}{R}
$$

where:

$-g_{m}$ is the transconductance of the NMOS transistor;

$-R$ is the resistive part of the resonator.

In these conditions, the sizes of NMOS transistors $T_{1}$ to $T_{4}$ are identical and chosen to be $\left(\frac{\mathrm{W}}{\mathrm{L}}\right)=\frac{70 \mu \mathrm{m}}{0.35 \mu \mathrm{m}}$.

The simulated tuning characteristic of one uncoupled VCO is presented in Fig. 5. As shown on this figure, the $\mathrm{VCO}$ is tuned from $5.55 \mathrm{GHz}$ to $7 \mathrm{GHz}$ with a tuning voltage varying from 0 to $2.5 \mathrm{~V}$.

Since the presented theory uses van der Pol oscillators to model microwave coupled oscillators, we performed the modelling of this structure as two differential van der Pol coupled oscillators as presented in [27], using ADS simulation results for one differential NMOS VCO at the required synchronization frequency. As a consequence, the two coupled VCOs of Fig. 4 can be reduced into two differential van der Pol coupled oscillators as shown in Fig. 6. In this case, let us note that the value of the coupling resistor on each path is equal to $R c / 2$ to match well with the theory based on the use of two single-ended van der Pol oscillators.

Hence, the passive part corresponding to the resonator of the VCO is modeled by a parallel $R L C$ circuit. Thus, they must have the same behavior, i.e. the same resonance frequency and the same quality factor, within the frequency band of operation of the VCO. This modeling was realized using a S-parameters simulation with ADS software, as shown in Fig. 7. Therefore, the resonator parameters of the Van der Pol oscillator were 
set so that the impedances presented by the VCO's resonator, $Z_{11}$, and the Van der Pol resonator, $Z_{22}$, will be equal. Hence, since the inductor value on each side is equal to $0.8 \mathrm{nH}$, the differential inductor of the Van der Pol resonator will be $L=1.6 \mathrm{nH}$. The resistor value, $R$, corresponds to the real part of $Z_{11}$ at the resonance frequency and was found to be equal to $260 \Omega$ as shown in Fig .8. The corresponding value of $G_{L}$ is thus $0.0038 \mathrm{~S}$. Then, the capacitor value $C$ was tuned so that $Z_{11}=Z_{22}$. Hence, for $C=0.456 \mathrm{pF}$, the real and imaginary part of the two impedances, $Z_{11}$ and $Z_{22}$ match well as illustrated in Fig 8.

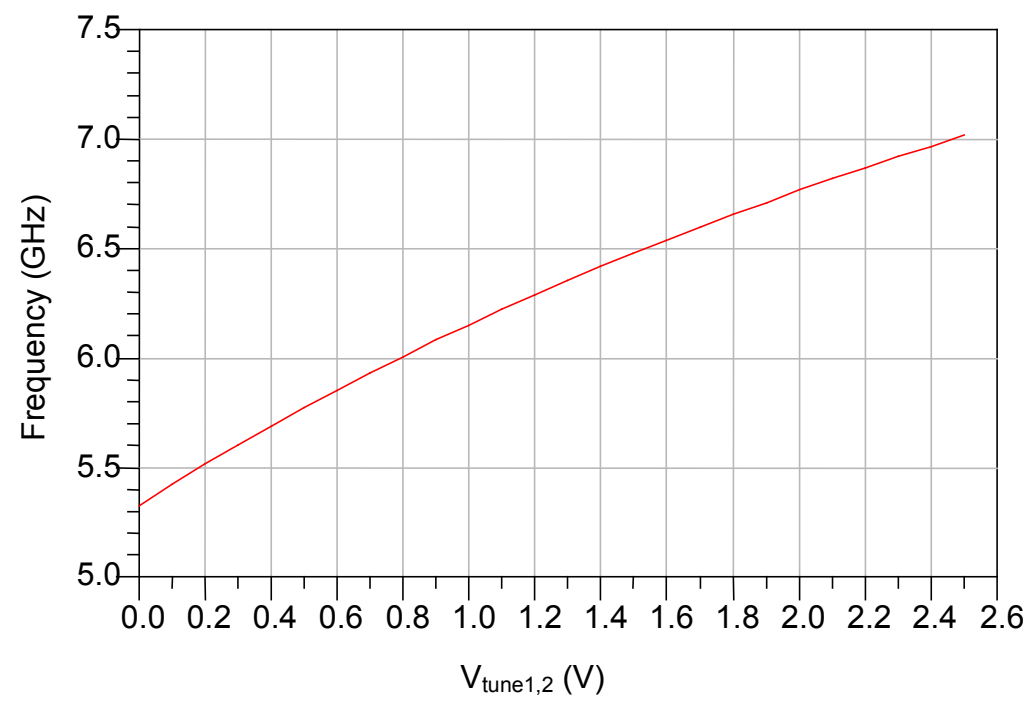

Figure 5: Tuning characteristic of the VCO

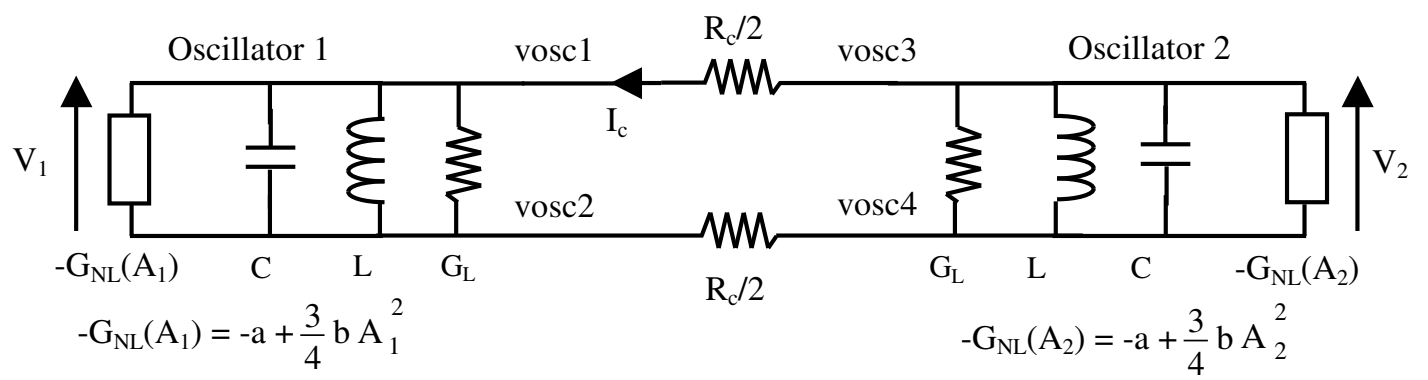

Figure 6: The two differential van der Pol coupled oscillators
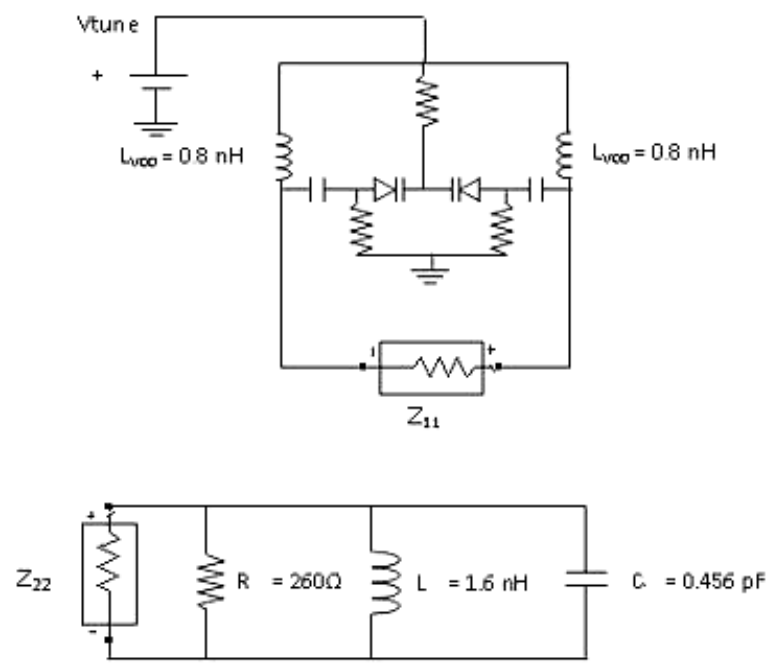

Figure 7: The identification of the parameters of the Van der Pol resonator 

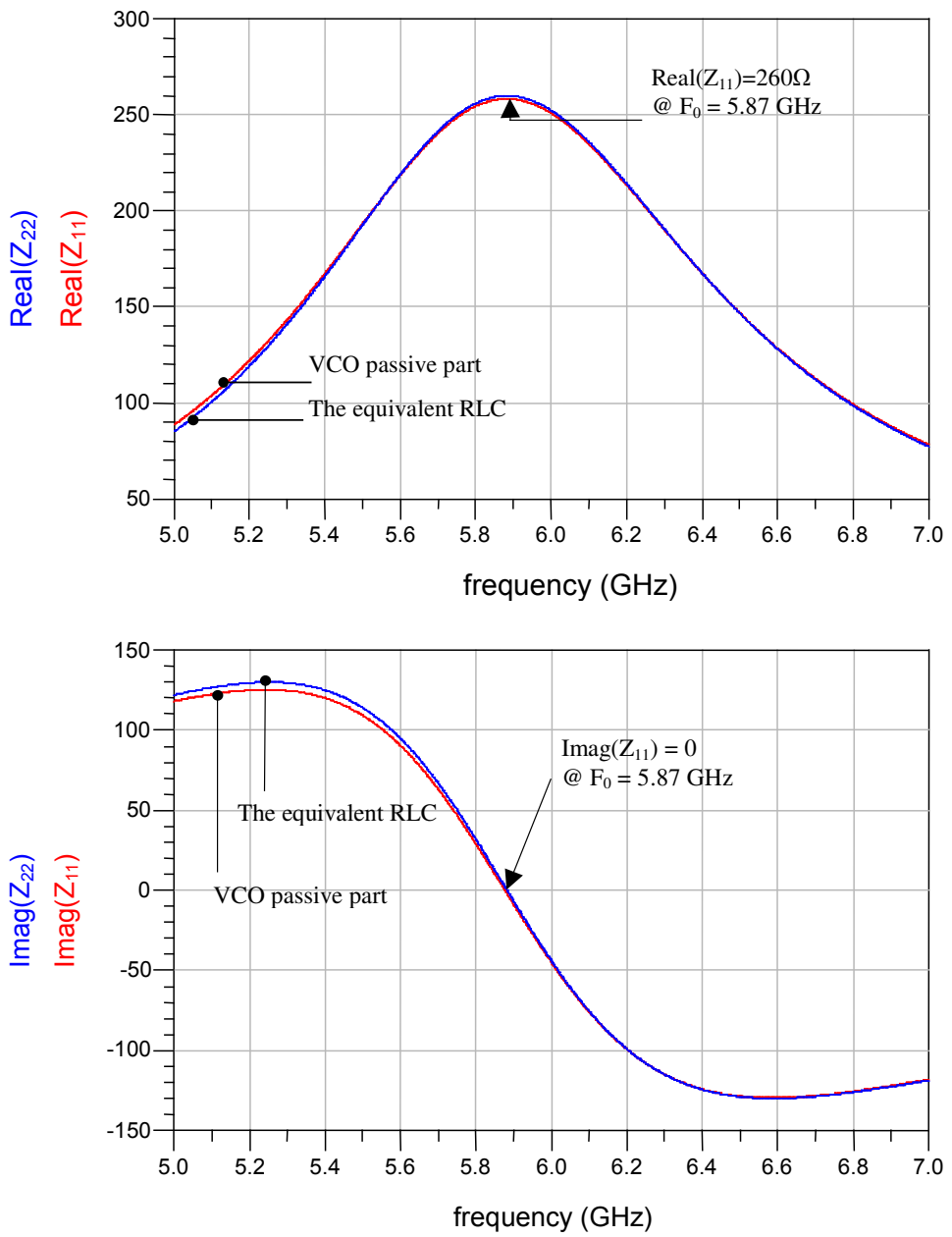

Figure 8: The real and imaginary part of the two impedances $Z_{11}$ and $Z_{22}$

For the modelling of the active part, the $I=f\left(V_{d i f f}\right)=f\left(V d_{I^{-}} V d_{2}\right)$ characteristic of one differential NMOS VCO of Fig.4 at the required synchronization frequency was performed leading to the typical cubic non linearity of a van der Pol oscillator as shown in Fig. 9.

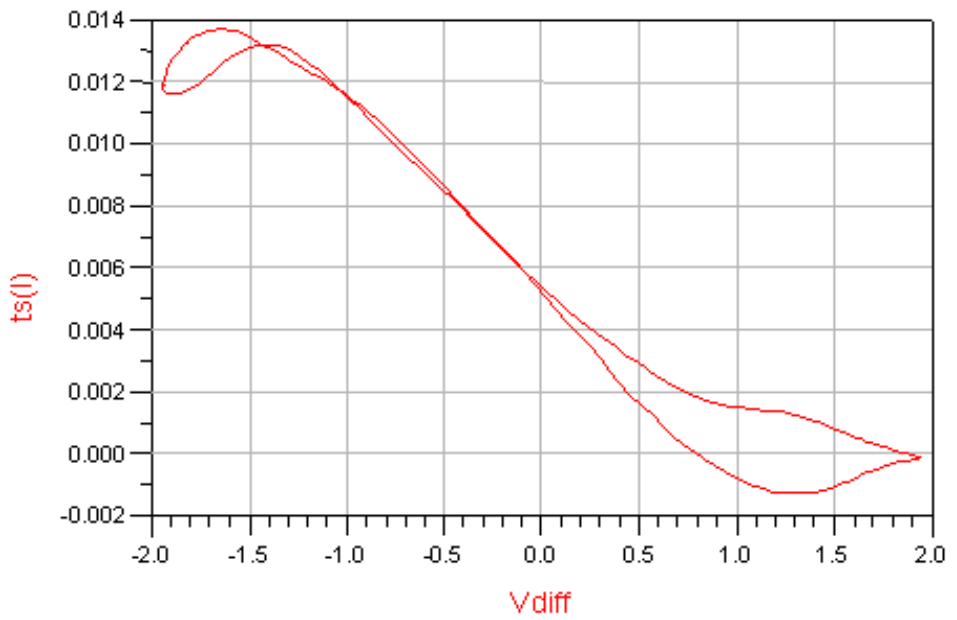

Figure 9: The cubic non linearity of a VCO 
Let us now remind the general expression of the current in a Van der Pol oscillator as already mentioned in section 2 :

$$
i=-a v_{d i f f}+b v_{d i f f}^{3}
$$

The parameter $a$ is given by the slope of the Van der Pol characteristic of Fig. 9 when $V_{\text {diff }}=0$, so that:

$$
a=\frac{\Delta I}{\Delta V_{\text {diff }}} \approx 0.00662
$$

Now according to the theory of a Van der Pol oscillator [28], the amplitude of the oscillation is equal to $2 \sqrt{\frac{a-G_{L}}{3 b}}$, with $G_{L}=\frac{1}{R}$.

Since the free-running amplitude of the differential voltage of one VCO of Fig. 4 at the required synchronization frequency is equal to $1.941 \mathrm{~V}$, we can deduce the value of parameter $b$ so that:

$$
\text { 2. } \sqrt{\frac{a-G}{3 b}}=1.941 \Rightarrow b=0.000976 .
$$

Now, using (2), the phase difference $\Delta \phi$ can be found for a given parameter $\Delta \omega_{0}$. For instance, if the free-running frequencies of the two oscillators are $f_{01}=6.18 \mathrm{GHz}$ and $f_{02}=5.59 \mathrm{GHz}$ leading to $\Delta \omega_{0}=-3.7 \times 10^{9} \mathrm{rad} / \mathrm{s}$, then, the phase shift value $\Delta \phi$ is $-61.7^{\circ}$ for $\lambda_{0}=\frac{1}{G_{L} R_{c}}=0.5$ and $\omega_{a}=\frac{G_{L}}{2 . C}=4.21 \times 10^{9} \mathrm{rad} / \mathrm{s}$. Furthermore, since the synchronization frequency is located between the two oscillators free-running frequencies, the amplitudes are equal and found from (15) as $A_{1}=A_{2}=A=1.55 \mathrm{~V}$. In these conditions, the coupled system of Fig. 6 simulated with ADS leads to two sinusoidal waves at a synchronization frequency of $5.84 \mathrm{GHz}$ with a phase shift equal to $-61.27^{\circ}$ and two equal amplitudes of 1.556 $\mathrm{V}$ as shown in Fig. 10.

Let us now compare the previous results with those obtained with the two identical differential coupled VCOs of Fig. 4 under the same simulation conditions. To do so, the tuning voltages $V_{\text {tunel }}$ and $V_{\text {tune2 }}$ have been adjusted in order to obtain the same free-running frequencies i.e. $f_{01}=6.18 \mathrm{GHz}$ and $f_{02}=5.59 \mathrm{GHz}$. In these conditions, the two differential NMOS coupled VCOs of Fig.4 simulated with ADS has lead to two sinusoidal waves at a synchronization frequency of $5.86 \mathrm{GHz}$, a phase shift of $-65.6^{\circ}$ and an amplitude close to $1.5 \mathrm{~V}$ at the output of each oscillator, as presented in Fig. 11. As a consequence, a good agreement was found between the theoretical prediction of the phase shift as well as of the amplitude and the simulation results of the two coupled differential VCOs.

In order to generalize these results, the VCOs' tunings are moved apart so that the synchronization frequency will remain halfway between. In these conditions, Fig.12.a shows a comparison between the theoretical amplitude calculated using (15) and the simulated amplitude for the two coupled differential van der Pol oscillators and for the two coupled differential NMOS VCOs as a function of $\Delta f_{0}=f_{02}-f_{01}$. Moreover, Fig.12.b shows a comparison between the theoretical phase shift calculated using (2) and the simulated phase shift always as a function of $\Delta f_{0}$. These results are also summarized in Table I. 


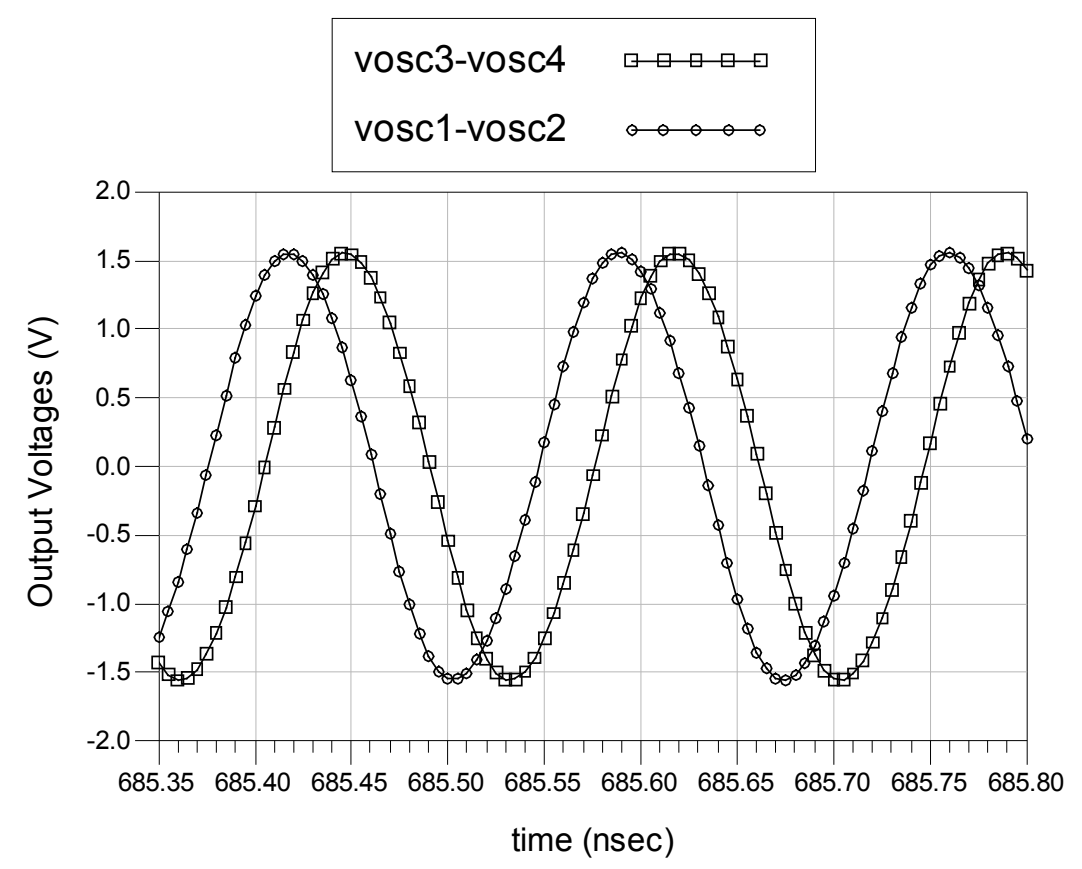

Figure 10: Waveforms of the output voltages of the two van der Pol oscillators for $\Delta \phi=-61.27^{\circ}$ and $A_{1}=A_{2}=A=1.556 \mathrm{~V}$

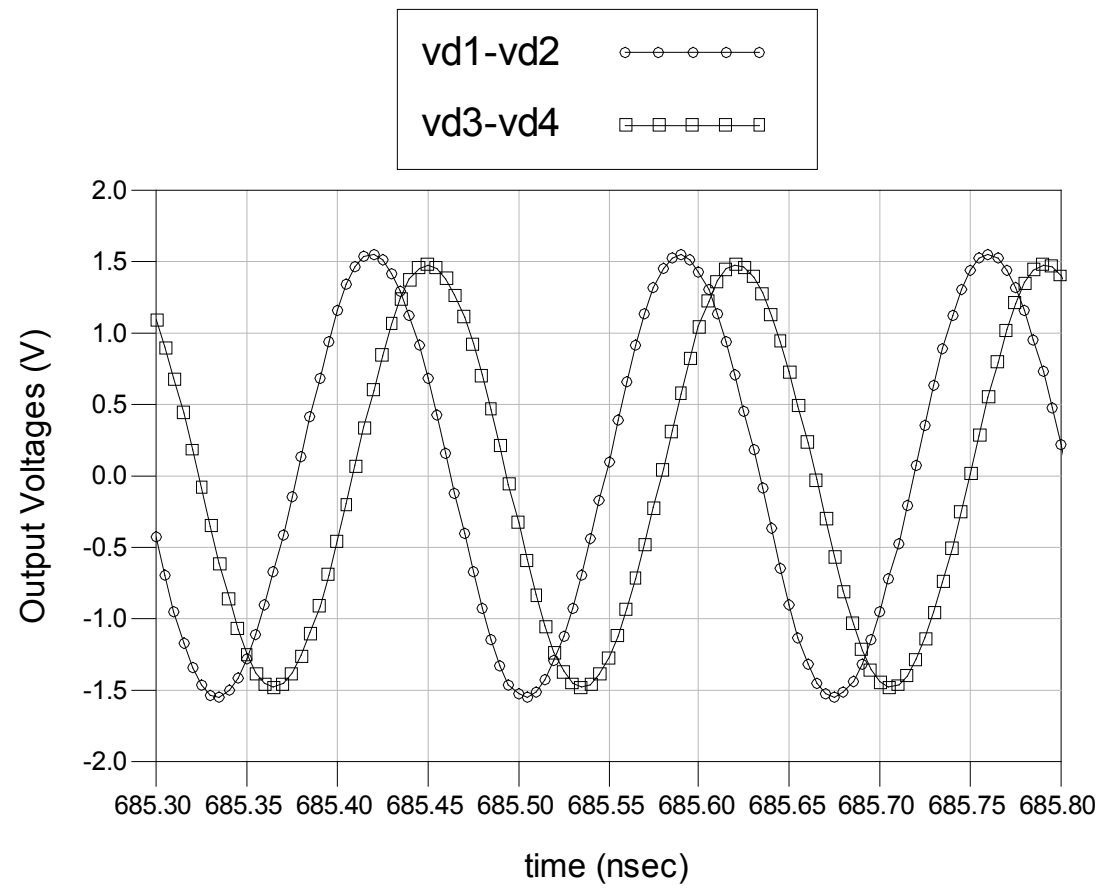

Figure 11: Waveforms of the output voltages of the two differential NMOS VCOs for $\Delta \phi=-65.6^{\circ}$ and $A \approx 1.5 \mathrm{~V}$ 


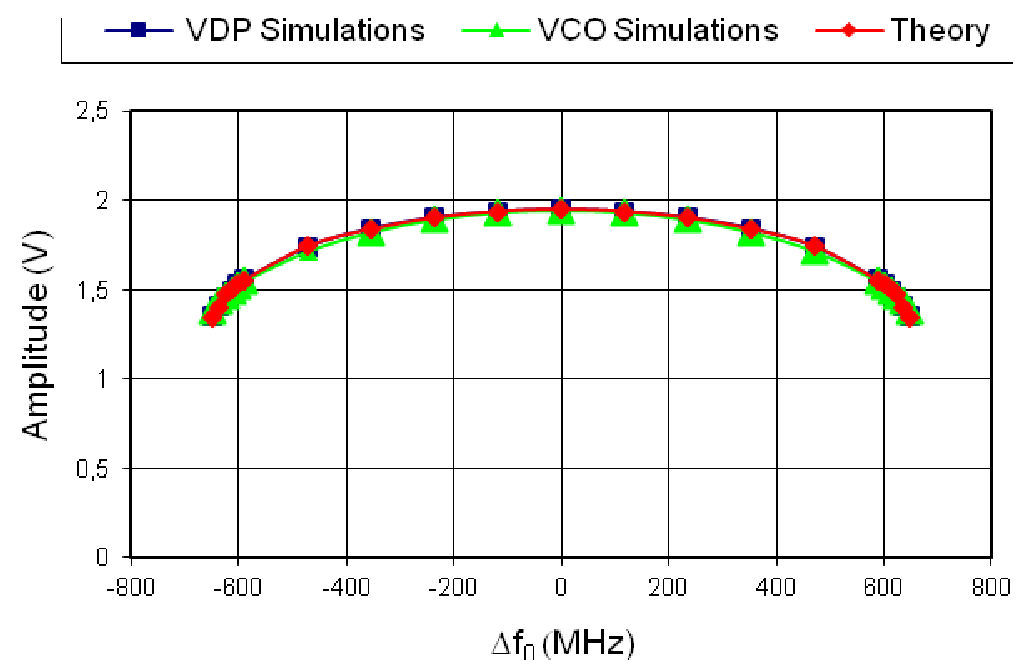

(a)

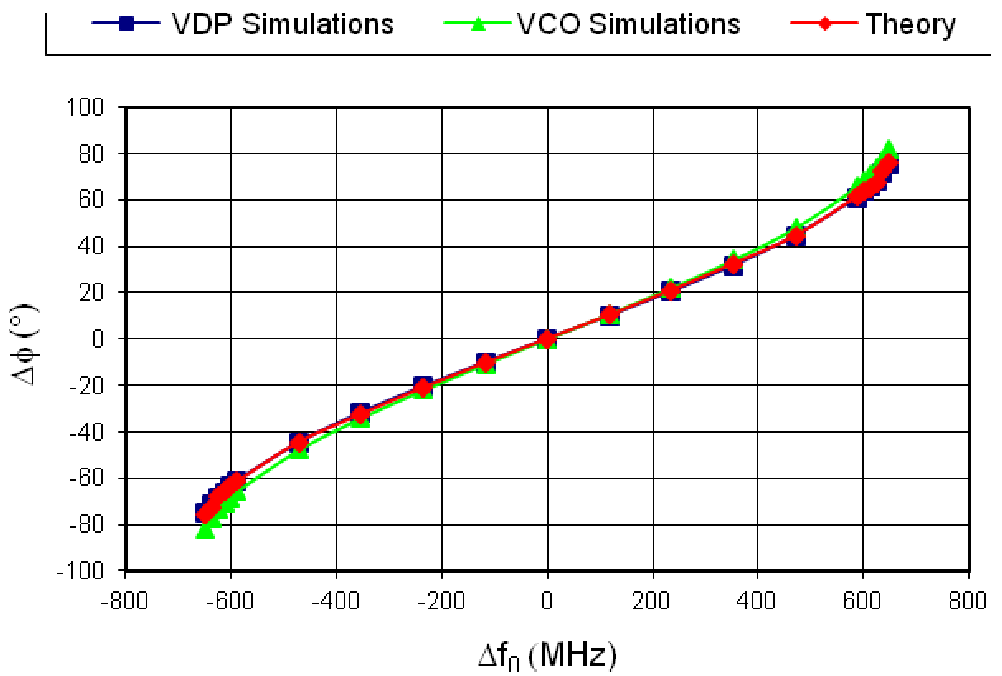

(b)

Figure 12: Comparison between the theoretical and simulated results for $(a)$ the amplitude and $(b)$ the phase shift

As can be seen on this figure, a good agreement is found between the theoretical prediction of the amplitude as well as of the phase shift and the simulation results over the entire range of VCOs' tunings. For the phase shift prediction, equation (2) was already found by J. Lynch and R. York in [19] for the case of a narrow-band coupling network and shows that, as the oscillator tunings are moved apart, but the synchronization frequency is halfway between, the phase shift increases until the locking-region boundary is encountered. This theoretical behaviour is verified in simulation as shown in Fig.12.b. Concerning the prediction of the amplitudes given by (15) and derived from the proposed system of equations found in (14), the amplitude is at a minimum at the locking-region boundaries (i.e. when the phase shift is at the extreme values) and equal to the oscillators' free-running amplitude for equal tunings (i.e. when the phase shift is equal to zero). As can be seen in Fig.12.a, this behaviour is also verified in simulation. Furthermore, these results show that the proposed theory can be used to design an array made of two differential VCOs coupled through a resistive network. Indeed, after the modelling step of one differential NMOS VCO at the required synchronization frequency, equations (2) and (15) can be used to adjust, with a relatively high accuracy, the free-running frequencies of the two differential NMOS VCOs required to achieve the desired phase shift and 
amplitudes. These equations can of course be used for others differential oscillators architectures and for a narrow-band coupling network.

Table I. Theoretical and simulated results summary for the amplitude and the phase shift

\begin{tabular}{|c|c|c|c|c|c|}
\hline \multirow{2}{*}{$\begin{array}{c}f_{\text {o1 }} \\
(G H z)\end{array}$} & $f_{o 2}$ & \multicolumn{2}{|c|}{ Amplitude $(V)$} & \multicolumn{2}{c|}{$\Delta \phi\left(^{\circ}\right)$} \\
\cline { 3 - 6 } & $(G H z)$ & $\begin{array}{c}\text { Simulations } \\
(V C O s)\end{array}$ & Theory & $\begin{array}{c}\text { Simulations } \\
(\text { VCOs })\end{array}$ & Theory \\
\hline 6.217 & 5.569 & 1.383 & 1.36 & -81.78 & -75.23 \\
\hline 6.205 & 5.58 & 1.47 & 1.458 & -73.61 & -68.7 \\
\hline 6.129 & 5.66 & 1.71 & 1.743 & -47.83 & -44.69 \\
\hline 6.07 & 5.72 & 1.82 & 1.845 & -34.03 & -31.83 \\
\hline 5.952 & 5.83 & 1.93 & 1.938 & -10.74 & -10.12 \\
\hline 5.89 & 5.89 & 1.94 & 1.95 & 0 & 0 \\
\hline 5.78 & 6.011 & 1.89 & 1.905 & 22.04 & 20.58 \\
\hline 5.66 & 6.129 & 1.71 & 1.743 & 47.83 & 44.69 \\
\hline 5.6 & 6.188 & 1.55 & 1.557 & 65.7 & 61.53 \\
\hline 5.59 & 6.2 & 1.5 & 1.496 & 70.78 & 66.1 \\
\hline 5.569 & 6.217 & 1.38 & 1.36 & 81.8 & 75.23 \\
\hline
\end{tabular}

\section{Conclusion}

A new formulation of the equations describing the locked states of two van der Pol oscillators coupled through a broadband network using an accurate model allowing a good prediction of the oscillators' amplitudes was presented in this paper. To do so, the system of nonlinear equations presented in [19] by J. Lynch and R. York and giving the dynamics for two van der Pol oscillators coupled through a resonant network was first overviewed to show the limitation of this theory regarding the prediction of the amplitudes of the two coupled oscillators. After that, the York's theory was adapted to the case of a more accurate van der Pol model in order to obtain the new system of four equations with four unknowns describing the locked states of two van der Pol oscillators coupled through a resonant network. The case of a resistive coupling circuit was then deduced from this system of equations. This has led to a new expression allowing the prediction of the amplitudes of the two coupled van der Pol oscillators for values of parameters $a$ and $b$ of the van der Pol non linearity leading to a sinusoidal waveform. The presented theory regarding the prediction of the oscillators' amplitudes was verified using Agilent's ADS software for the practical case of an array made of two differential VCOs coupled through a resistive network. The obtained results are consistent with the theory showing the usefulness of such a theoretical approach for the design of an array of differential VCOs coupled through a resistive network.

\section{References}

1. S. Nogi, J. Lin, and T. Itoh, "Mode analysis and stabilization of a spatial power combining array with strongly coupled oscillators", IEEE Trans.Microw. Theory Tech., 41 (10) (1993), 1827-1837.

2. M. R. Kuhn and E. M. Biebl, "Power combining by means of harmonic injection locking", in IEEE MTT-S Int. Microw. Symp. Dig, 2004, pp. 91-94.

3. T. Health, "Beam steering of nonlinear oscillator arrays through manipulation of coupling phases", IEEE Trans. Antennas Propag, 52 (7) (2004), 1833-1842.

4. P. Liao and R. A. York, "A new phase-shifterless beam-scanning technique using arrays of coupled oscillators", IEEE Trans. Microw. Theory Tech., 41 (10) (1993), 1810-1815.

5. N. Y. Tohmé, J.M. Paillot, D. Cordeau, P. Coirault, "Analysis of the Frequency Locking Region of Coupled Oscillators Applied to 1-D Antenna Arrays," European Microwave Conference, Amsterdam, 2008, pp. 1334-1337. 
6. R. A. York, "Nonlinear analysis of phase relationships in quasioptical oscillator arrays", IEEE Trans.Microw. Theory Tech, 41 (10) (1993), 1799-1809.

7. P. Liao and R. A. York, "Beam Scanning With Coupled VCOs", In Proc. Antennas Propagation Soc. Int. Symp., 1994, pp. 836-839.

8. R. A. York and T. Itoh, "Injection and phase-locking techniques for beam control", IEEE Trans.Microw. Theory Tech, 46 (11) (1998), 1920-1929.

9. Y. Uwate, Y. Nishio and R. Stoop, "Complex pattern in a ring of van der Pol oscillators coupled by time-varying resistors", Journal of Circuits, Systems and Computers, 19 (4) (2010), 819-834.

10. D. Aoun and D. A. Linkens, "Computer-Aided Mode Analysis of Coupled Nonlinear Oscillators", IEEE Trans. Circuits Syst., 37 (2) (1990), 273-280.

11. Chai Wah Wu and Leon O. Chua, "Application of Graph Theory to the Synchronization in an Array of Coupled Nonlinear Oscillators", IEEE Trans. Circuits Syst. I, 42 (8) (1995), 494-497.

12. D. Kuroki, T. Endo and S. Mori, "An analysis of oscillation modes of four completely coupled oscillators via integral manifolds and its experimental confirmation", Electronics and Communications in Japan. III, 81 (9) (1998), 20-28.

13. V. Lanza, F. Corinto, M. Gilli and P. P. Civalleri, "Analysis of nonlinear oscillatory network dynamics via timevarying amplitude and phase variables", Int. J. Circuit Theory Appl., 35 (5-6) (2007), 623-644.

14. P. Maffezzoni, "Synchronization Analysis of Two Weakly Coupled Oscillators Through a PPV Macromodel", IEEE Trans. Circuits Syst. I, 57 (3) (2010), 654-663.

15. A. Buonomo and A. Lo Schiavo, "The effect of parameter mismatches on the output waveform of an LC-VCO", Int. J. Circuit Theory Appl., 38 (5) (2010), 487-501.

16. R. A. York and P. Liao, "Oscillator Array Dynamics with Broadband N-Port Coupling Networks", IEEE Trans.Microw. Theory Tech, 42 (11) (1994), 2040-2045.

17. H-C Chang, X. Cao, U. K. Mishra and R. A. York, "Phase Noise in Coupled Oscillators: Theory and Experiment" IEEE Trans.Microw. Theory Tech, 45 (5) (1997), 604-615.

18. K. Kurokawa, "Injection locking of solid state microwave oscillators", In proc. IEEE, 61 (10) (1973), 1386-1409.

19. J. J. Lynch and R. A. York, "Synchronization of Oscillators Coupled Through Narrow-Band Networks," IEEE Trans.Microw. Theory Tech, 49 (2) (2001), 237-249.

20. A. Collado, F. Ramirez, A. Suarez and J. P. Pascual, "Harmonic-Balance Analysis and Synthesis of CoupledOscillator Arrays" IEEE Microw. Wireless Compon. Lett., 14 (5) (2004), 192-194.

21. A. Suarez, A. Collado and F. Ramirez, "Harmonic-Balance techniques for the design of coupled-oscillator systems in both unforced and injection-locked operation" in IEEE MTT-S Int. Microw. Symp. Dig, 2005, pp. 887-890.

22. A. Georgiadis, A. Collado and A. Suarez, "New Techniques for the Analysis and Design of Coupled-Oscillator Systems" IEEE Trans.Microw. Theory Tech, 54 (11) (2006), 3864-3877.

23. A. Suarez, Analysis and Design of Autonomous Microwave Circuits. (Wiley-IEEE press ; 2009).

24. A. Suarez, F. Ramirez and S. Sancho, "Stability and Noise Analysis of Coupled-Oscillator Systems" IEEE Trans.Microw. Theory Tech, 59 (4) (2011), 1032-1046.

25. Odyniec M, RF and microwave oscillator design. (Boston, MA: Artech House; 2002).

26. A. Hajimiri and T. H. Lee, "Design Issues in CMOS Differential LC Oscillators", IEEE Journal of Solid-State Circuits, 34 (5) (1999), 717-724.

27. M.I. Ionita, D. Cordeau, J.M. Paillot, M. Iordache, "Analysis and Design of an Array of Two Differential Oscillators Coupled Through a Resistive Network", European Conference on Circuit Theory and Design, 2011, pp. 73-76.

28. B. Van der Pol, "The nonlinear theory of electric oscillations", Proceedings of the IRE, 22 (9) (1934), 1051-1086. 